\title{
A New Extension Exponential Distribution with Applications of COVID-19 Data
}

\author{
Amaal El Sayed Abd El Ghany Mubarak \\ Assistant Professor, Department of Applied, mathematical and actuarial \\ Statistics, Faculty of Commerce - Damietta University, Egypt \\ prof_amaal2010@yahoo.com
}

\section{Ehab M. Almetwally}

Assistant lecturer, Faculty of Business Administration, Delta University of Science and Technology, Egypt

ehabxp_2009@hotmail.com 


\begin{abstract}
In this paper, we introduce and study a new extension of exponential distribution with three-parameter named as the X-Gamma extension of the exponential (XGExEx) distribution. Survival function and hazard function for XGExEx distribution are discussed. Maximum likelihood estimation (MLE) and maximum product spacing (MPS) Methods for the XGExEx distribution parameters are discussed. A numerical study using real data analysis and Monte-Carlo simulation are performed to compare between different methods of estimation. Superiority of the new model over some well-known distributions are illustrated by COVID-19 Data. The XGExEx model can produce better fits than some well-known distributions as the Marshall-Olkin alpha power exponential (MOAPE) [Nassar et al. (2019)], extended odd Weibull exponential (EOWE) [Afify and Mohamed (2020)], WeibullExponential (WE) [Oguntunde et al. (2015)], ExEx and generalized exponential (GE) [Gupta and Kundu (2001)].
\end{abstract}

Keywords: X-Gamma; exponential distribution; maximum likelihood estimation; maximum product spacing; and COVID-19 data analysis. 
مجلة البحوث المالية والتجارية ـ المجلا (22) - العدد الأول - يناير 2021

\section{الملخص باللغة العربية}

\section{امتداد جديد للتوزيع الأسي مع التطبيق علي بيانات 19-COVID}

في هذا البحث ، قدمنا ودرسنا امتدادًا جديدًا للتوزيع الأسي بثلاثة معلمات، ومن الممكن تسميته X-Gamma extension of the exponential للتوزيع الأستاد X-Gamma ويمكن ان يطلق عليه الاختصار التالي XGExEx. تمت مناقثة دالة البقاء ودالة الخطر لتوزيع الأليع

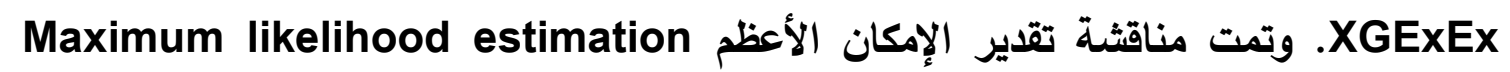

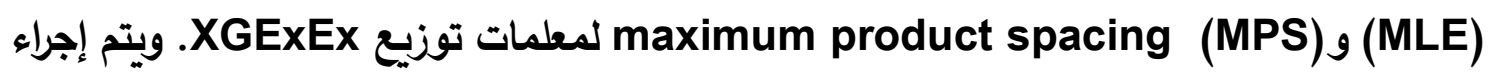
دراسة عددية باستخدام تحليل البيانات الحقيقية ومحاكاة مونت كارلو للمقارنة بين طرق التقدير

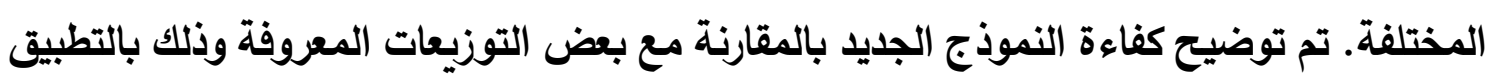
على بيانات COVID-19. واتضح أن نموذج XGExEx أفضل ملاءمة من بعض التوزيعات

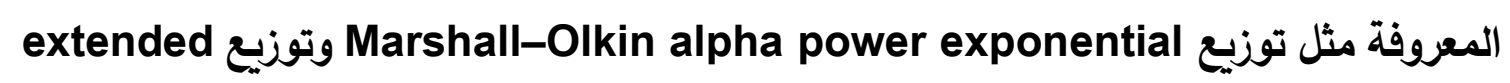
odd Weibull امتداد الأسي extension of the exponential والتوزيع الأسي المعم generalized exponential

كنلك تم تقييم مرونة واعتمادية توزيع (XGExEx) المقترح من خلال مجموعتين من البيانات الحقيقية لفيروس كورونا المستحدث، وذلك باستخدام بيانات يومية لمعدل الوفيات الخام لدولة مئل

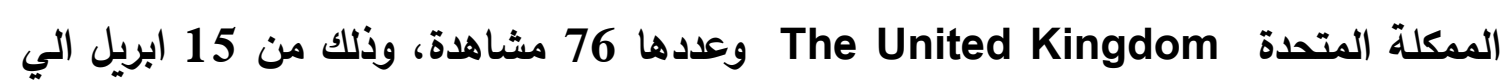
30 يونيو 2020. أما مجموعة البيانات الأخرى فهي بيانات يومية عن معدل الوفيات الخام لدولة

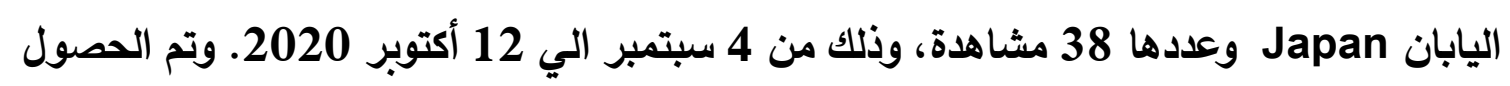
على هذه البيانات من إصدارات منظمة الصحة العالمية.

الكلمات المفتاحية: عائلة X-Gamma، توزيع extension of the exponential، طريقة الامكان الأعظم، maximum product spacing، تحليل البيانات 


\section{Introduction}

The corona virus disease (COVID-19) is considered one of the worst pandemics in the recent years. Most countries have increased their health measures to decrease the spread rate of the COVID-19 by postponing education, closing borders and Appling partial curfew rules.

While Medical Researchers have spent lots of time to find medical solutions to return to normal life, other researchers have focused on the mathematical and statistical modeling of the COVID-19 outbreak to make future predictions. Remuzzi and Remuzzi (2020) predicted how many hospital beds and personnel Italy needed under the exponential trend. Maleki et al. (2020) forecasted the recovered and confirmed COVID-19 cases using autoregressive time series models based on the two-piece scale mixture normal distributions. Caccavo (2020) introduced the compartmental susceptible-infectious-recovereddeceased (SIRD) model differentiates between recovered and diseased cases within each compartment and predict COVID-19 cases in China and Italy. Ayyoubzadeh et al. (2020) predicted COVID-19 cases in Iran by using long -short term memory (LSTM) which is a deep-learning method. El-Morshedy et al. (2020) introduced a new discrete analogous of the generalized Lindley Distribution to model the daily new cases and deaths of COVID-19. Almetwally et al. (2020) introduced a new discrete analogous of the discrete Marshall-Olkin generalized exponential distribution to model the daily new cases and deaths of COVID-19 data. Almetwally and Gamal (2020) introduced a new discrete analogous discrete alpha power inverse Lomax distribution to analyze the daily count of new cases of the COVID-19 of Australia.

The extension of the exponential (ExEx) distribution was introduced by Nadarajah and Haghighi (2011). The cumulative distribution function (CDF) and the probability density function (PDF) of the ExEx distribution are respectively as follows

$$
\begin{gathered}
F(x ; \varphi, \omega)=1-e^{\left[1-(1+\omega x)^{\varphi}\right]} ; \quad x>0, \varphi, \omega>0, \\
f(x ; \varphi, \omega)=\omega \varphi(1+\omega x)^{\varphi-1} e^{\left[1-(1+\omega x)^{\varphi}\right]} .
\end{gathered}
$$

Modified and extended versions of the ExEx distribution have been studied by many authors as Gómez et al. (2014) presented a new extension of the exponential distribution based on mixtures of positive distributions. Khan et al. (2015) studied transmuted generalized exponential distribution for analyzing lifetime data. Muhammad (2016) introduced a new family of distributions called the Poisson-odd generalized exponential distribution. De Andrade et al. (2016) introduced exponentiated generalized extended exponential 
distribution. Kumar et al. (2017) discussed extended exponential distribution based on order statistics. Hassan et al. (2018) proposed a new distribution called the alpha power transformed extended exponential distribution. Pena-Ramirez et al. (2018) proposed a new lifetime model called the exponentiated power generalized Weibull distribution, which is obtained from the exponentiated family applied to the power generalized Weibull distribution.

During recent years, the X-Gamma (XG) distribution has been shown great interest by researchers, which is introduced by Sen et al. (2016). The quasi-XG distribution has been introduced by Sen and Chandra (2017). A new bounded distribution has been introduced by Altun and Hamedani (2018) using the transformation $Y=e^{-X}$ as an alternative to the beta distribution based on the XG distribution. Another generalization of XG distribution has been provided by Sen et al. (2018a) on the basis of a special mixture of exponential and gamma distributions. Parameter estimation of XG distribution under the progressively type-II censored sample has been studied by Sen et al. (2018b) by using different methods. The transmuted-XG distribution has been studied by Biçer (2019). Yadav et al. (2019) introduced inverse $X$-Gamma distribution using the transformation $Y=\frac{1}{X}$. Bantan et al. (2020) introduced the half-logistic XG distribution using half-logistic family. Sen et al. (2020) studied discrimination analysis between the Lindley and XG distribution.

On the other hand, the XG-Generator (XG-G) family has been proposed by Cordeiro et al. (2019) to incorporate any distribution into a larger family. The XG-G family has flexible shapes to model various lifetime data sets. The XG-G family add a parameter with one extra shape parameter $\boldsymbol{\vartheta}>\mathbf{0}$, the CDF of XG-G familyis given by

$$
\begin{aligned}
F(x ; \vartheta, \psi)=1 & -\frac{[1-G(x ; \psi)]^{\vartheta}}{\vartheta+1}\{1+\vartheta-\vartheta \ln (1-G(x ; \psi)) \\
& \left.+0.5 \vartheta^{2}[\ln (1-G(x ; \psi))]^{2}\right\},
\end{aligned}
$$

where $G(x ; \psi)$ is a baseline cdf with a parameter vector $\psi$. The PDF of XG-G family can be expressed as

$$
f(x ; \vartheta, \psi)=\frac{\boldsymbol{\vartheta}}{\boldsymbol{\vartheta}+\mathbf{1}} \boldsymbol{g}(\boldsymbol{x} ; \boldsymbol{\psi})[\mathbf{1}-\boldsymbol{G}(\boldsymbol{x} ; \boldsymbol{\psi})]^{\vartheta-1}\left\{\boldsymbol{\vartheta}+0.5 \boldsymbol{\vartheta}^{2}[\ln (1-G(x ; \psi))]^{2}\right\}
$$

where $g(x ; \psi)=d G(x ; \psi) / d x$.

The aim of this paper is to clarify two things. Firstly, propose and study a new lifetime distribution called $X$-Gamma extension of the exponential (XGExEx) distribution based on the XG-G family. Some statistical properties of XGExEx distribution are provided. Secondly, 
parameters estimation for the XGExEx distribution are discussed by using MLE and MPS methods. To evaluate the performance of the estimators, extensive simulation study is carried out. Our XGExEx model as well as some other well-known distributions are illustrated by COVID-19 Data. The XGExEx distribution can produce better fits than these other distributions.

The paper is organized as follows; in section 2, we introduce the description, notation and the statistical properties of XGExEx distribution. In section 3 we discuss the parameter estimation of XGExEx distribution. In section 4, Monte-Carlo simulation study are presented to compare the performance of the parameter's estimation for different methods. In section 5, application of COVID-19 Data is studied. Lastly in section 6, we discuss the results and conclusions of the current study.

\section{Model Description and Notation}

In this section, we will introduce the XGEXE distribution and some of its sub-models.

\subsection{XGExEx Distribution}

The XG-G family and ExEx distribution have been used to generate XGExEx distribution. It is represented by the random variable $X \sim$ $X G \operatorname{Ex} \operatorname{Ex}(\vartheta, \varphi, \omega)$. By using Equations (1.3, 1.4, 1.1 and 1.2), the CDF of XGExEx distribution takes this form

$$
F(x ; \vartheta, \varphi, \omega)=1-\frac{e^{\vartheta\left[1-(1+\omega x)^{\varphi}\right]}}{\vartheta+1}\left\{1+(1+\omega x)^{\varphi}\left(\vartheta-\vartheta^{2}\right)+0.5 \vartheta^{2}\left[1+(1+\omega x)^{2 \varphi}\right]\right\},
$$

where $\vartheta, \varphi, \omega>0$ and $x>0$. The PDF of XGL distribution is given as:

$$
f(x, \vartheta, \varphi, \omega)=\frac{\vartheta}{\vartheta+1} \omega \varphi(1+\omega x)^{\varphi-1} e^{\vartheta\left[1-(1+\omega x)^{\varphi}\right]}\left\{\vartheta+0.5 \vartheta^{2}\left[1-(1+\omega x)^{\varphi}\right]^{2}\right\}
$$

Figure 1 display plots of the PDF of the XGExEx distribution for some parameters values as follows
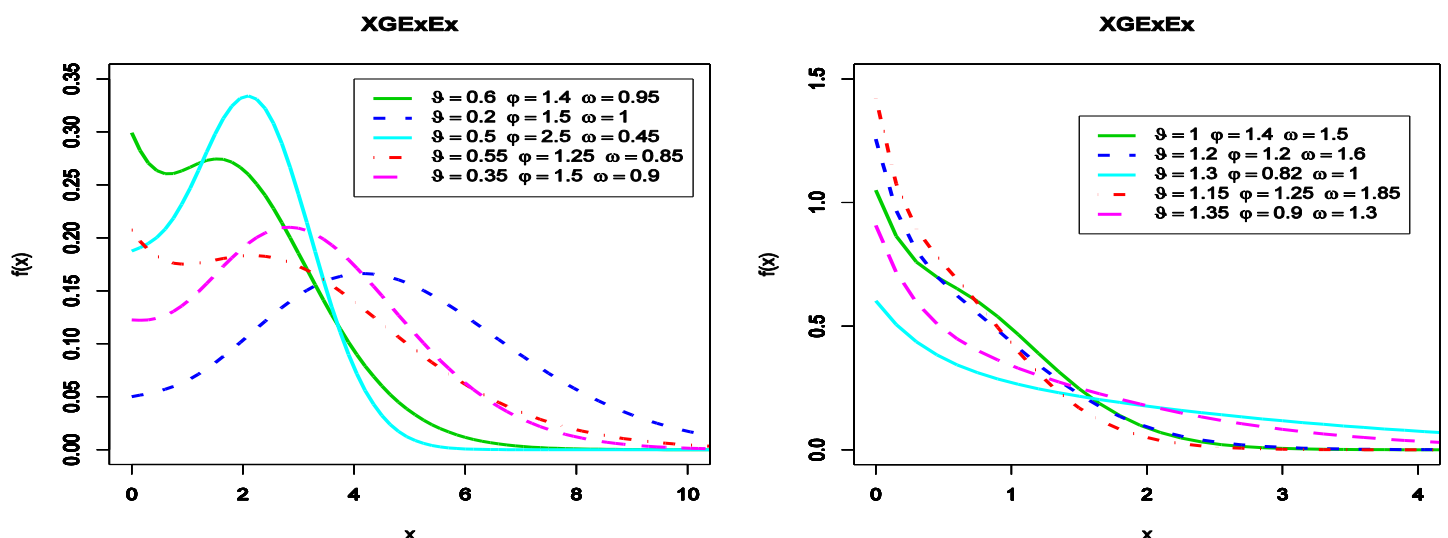

Figure 1. Plots of the PDF of the XGExEx distribution with Some Values of Parameters. 
Therefore, a random variable with PDF (2.2) is denoted by X XGExEx $(\vartheta, \varphi, \omega)$ reduces to the two parameters, this is a new model can be denoted as X-Gamma exponential (XGEx) distribution when $\quad \varphi \rightarrow 1$.

\subsection{Reliability functions of XGExEx distribution}

The survival function of XGExEx distribution is given by

$$
S(x ; \vartheta, \varphi, \omega)=\frac{e^{\vartheta\left[1-(1+\omega x)^{\varphi}\right]}}{\vartheta+1}\left\{1+(1+\omega x)^{\varphi}\left(\vartheta-\vartheta^{2}\right)+0.5 \vartheta^{2}\left[1+(1+\omega x)^{2 \varphi}\right]\right\}
$$

The hazard function of a lifetime random variable $X$ with XGExEx distribution is given by

$$
h(x ; \vartheta, \varphi, \omega)=\frac{\vartheta \omega \varphi(1+\omega x)^{\varphi-1}\left\{\vartheta+0.5 \vartheta^{2}\left[1-(1+\omega x)^{\varphi}\right]^{2}\right\}}{\left\{1+(1+\omega x)^{\varphi}\left(\vartheta-\vartheta^{2}\right)+0.5 \vartheta^{2}\left[1+(1+\omega x)^{2 \varphi}\right]\right\}}
$$

Figure 2 display plots of the hazard function of the XGExEx distribution for some values of the parameters as follows
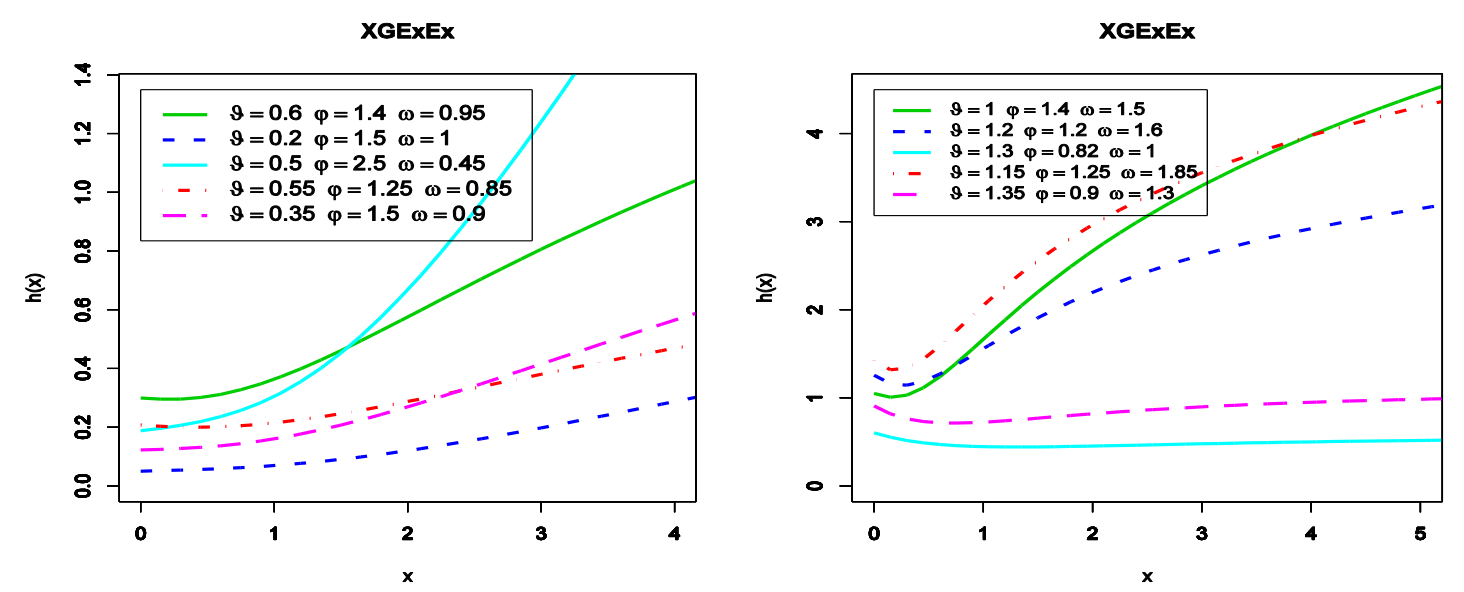

Figure 2. Plots of the hazard of the XGExEx with Some Values of the Parameters.

\section{Parameter Estimation}

In this section, the parameter estimation for the XGExEx distribution using MLE and MPS methods will be discussed in details.

\subsection{MLE method}

The log-likelihood function of XGExEx distribution, is given by:

$$
\begin{aligned}
l(\vartheta, \varphi, \omega)=n & \ln \left(\frac{\vartheta}{\vartheta+1}\right)+n[\ln (\varphi)+\ln (\omega)]+n \vartheta-\sum_{i=1}^{n}\left(1+\omega x_{i}\right)^{\varphi} \\
& +(\varphi-1) \sum_{i=1}^{n} \ln \left(1+\omega x_{i}\right)+\sum_{i=1}^{n} \ln \left\{\vartheta+0.5 \vartheta^{2}\left[1-\left(1+\omega x_{i}\right)^{\varphi}\right]^{2}\right\}
\end{aligned}
$$

Equation (3.1) can be maximized directly by using the $R$ package with an optimization function, to solve the non-linear likelihood equations 
obtained by differentiating Equation (3.1) with respect to $\vartheta, \varphi, \omega$ and equating to zero. The non-linear likelihood equations are given as

$$
\begin{aligned}
\frac{\partial l(\vartheta, \varphi, \omega)}{\partial \vartheta}= & \frac{n}{\vartheta(\vartheta+1)}+n+\sum_{i=1}^{n} \frac{1+\vartheta\left[1-\left(1+\omega x_{i}\right)^{\varphi}\right]^{2}}{\vartheta+0.5 \vartheta^{2}\left[1-\left(1+\omega x_{i}\right)^{\varphi}\right]^{2}} \\
\frac{\partial l(\vartheta, \varphi, \omega)}{\partial \varphi}= & \frac{n}{\varphi}-\sum_{i=1}^{n}\left(1+\omega x_{i}\right)^{\varphi} \ln \left(1+\omega x_{i}\right)+\sum_{i=1}^{n} \ln \left(1+\omega x_{i}\right) \\
& +\sum_{i=1}^{n} \frac{\vartheta^{2}\left[1-\left(1+\omega x_{i}\right)^{\varphi}\right] \ln \left(1+\omega x_{i}\right)}{\vartheta+0.5 \vartheta^{2}\left[1-\left(1+\omega x_{i}\right)^{\varphi}\right]^{2}}
\end{aligned}
$$

and

$$
\begin{aligned}
\frac{\partial l(\vartheta, \varphi, \omega)}{\partial \omega}= & \frac{n}{\omega}-\varphi \sum_{i=1}^{n} x_{i}\left(1+\omega x_{i}\right)^{\varphi-1}+(\varphi-1) \sum_{i=1}^{n} \frac{x_{i}}{1+\omega x_{i}} \\
& +\vartheta^{2} \varphi \sum_{i=1}^{n} x_{i} \frac{\left[1-\left(1+\omega x_{i}\right)^{\varphi}\right]\left(1+\omega x_{i}\right)^{\varphi-1}}{\vartheta+0.5 \vartheta^{2}\left[1-\left(1+\omega x_{i}\right)^{\varphi}\right]^{2}}
\end{aligned}
$$

\subsection{MPS Method}

MPS method is used to estimate the parameters of continuous univariate models as an alternative to the MLE method. The uniform spacings of a random sample $x_{1}<\cdots<x_{n}$ of size $n$ from the XGExEx distribution can be defined by

$$
D_{i}(\vartheta, \varphi, \omega)=F\left(x_{i} ; \vartheta, \varphi, \omega\right)-F\left(x_{i-1} ; \vartheta, \varphi, \omega\right) ; i=1,2, \ldots, n+1
$$

where $D_{i}$ denotes to the uniform spacings and $\sum_{i=1}^{n+1} D_{i}=1$. The MPS estimators can be obtained by maximizing

$$
G(\vartheta, \varphi, \omega)=\frac{1}{n+1} \sum_{i=1}^{n+1} \ln \left(D_{i}(\vartheta, \varphi, \omega)\right)
$$

To more information of MPS method, see Cheng and Amin (1983) and Almetwally and Almongy $\left(\mathbf{2 0 1 9}_{\mathrm{b}, \mathrm{a}}\right)$.

The natural logarithm of the product spacing function of XGExEx distribution is given by

$$
\begin{aligned}
\ln G(\vartheta, \varphi, \omega) & =\frac{1}{n+1}\left(\left[\ln \left(1-b\left(x_{1} ; \vartheta, \varphi, \omega\right)\right)-\ln \left(b\left(x_{n} ; \vartheta, \varphi, \omega\right)\right)\right]\right. \\
& \left.+\ln \left(b\left(x_{i-1} ; \vartheta, \varphi, \omega\right)-b\left(x_{i} ; \vartheta, \varphi, \omega\right)\right)\right)
\end{aligned}
$$

Where $b\left(x_{i}, \vartheta, \varphi, \omega\right)=\frac{e^{\vartheta\left[1-\left(1+\omega x_{i}\right)^{\varphi}\right]}}{\vartheta+1}\left\{1+\left(1+\omega x_{i}\right)^{\varphi}\left(\vartheta-\vartheta^{2}\right)+0.5 \vartheta^{2}\left[1+\left(1+\omega x_{i}\right)^{2 \varphi}\right]\right\}$.

The partial derivatives of MPS with respect to the unknown parameters cannot be solved explicitly, so numerical methods like the conjugate gradients algorithms can be used to calculate the MPS of $\vartheta, \varphi, \omega$. 
مجلة البحوث المالية والتجارية ـ المجلد (22) ـ العدد الأول - يناير 2021

\section{Simulation Study}

In this section; a Monte Carlo simulation is done to estimate the parameters by using MLE and MPS methods. R packages were used and the Simulation algorithm was as follows:

Monte Carlo experiments were carried out based on 1000 random sample for following data generated form XGExEx distribution by using numerical analysis in R packages, where $x_{i}$ is distributed as XGExEx distribution for different parameters $\Omega=(\vartheta, \varphi, \omega)$ with different actual values of parameter and for different samples sizes $n=35,75$ and 150. We can find the parameter estimation by using Equations ( 3.1 and 3.2) and an optim function in $R$ packages. We could define the best method as which minimizes the Bias and mean squared error (MSE) of the estimator. From Tables (1-2) we can see that all the estimates reveal the property of consistency, the Bias and MSE decrease when $\boldsymbol{n}$ increase. Also, can see that the MPS method has more relative efficiency than MLE. 
A New Extension Exponential Distribution with Applications of COVID-19 Data. (Dr.Amaal El Sayed Mubarak\& Ehab M. Almetwally)

Table 1: MLE and MPS estimation methods for XGExEx distribution with different values of parameters when $\vartheta=0.5$

\begin{tabular}{|c|c|c|c|c|c|c|c|c|c|c|c|c|c|c|}
\hline \multirow{2}{*}{\multicolumn{2}{|c|}{$\vartheta=0.5$}} & \multirow{3}{*}{$\varphi$} & \multicolumn{4}{|c|}{0.5} & \multicolumn{4}{|c|}{1.4} & \multicolumn{4}{|c|}{2.2} \\
\hline & & & \multicolumn{2}{|c|}{ MLE } & \multicolumn{2}{|c|}{ MPS } & \multicolumn{2}{|c|}{ MLE } & \multicolumn{2}{|c|}{ MPS } & \multicolumn{2}{|c|}{ MLE } & \multicolumn{2}{|c|}{ MPS } \\
\hline$\omega$ & $\mathrm{n}$ & & Bias & MSE & Bias & MSE & Bias & MSE & Bias & MSE & Bias & MSE & Bias & MSE \\
\hline \multirow{9}{*}{0.5} & \multirow{3}{*}{35} & $\vartheta$ & -0.0007 & 0.1385 & 0.0425 & 0.0218 & -0.0389 & 0.2070 & -0.0100 & 0.0315 & -0.0330 & 0.1778 & 0.0326 & 0.0385 \\
\hline & & $\varphi$ & 0.0227 & 0.0786 & -0.0194 & 0.0052 & 0.1461 & 0.2963 & -0.0216 & 0.0853 & 0.1016 & 0.2790 & -0.0493 & 0.0421 \\
\hline & & $\omega$ & -0.0131 & 0.0906 & 0.0421 & 0.0093 & 0.0561 & 0.2759 & 0.1506 & 0.1189 & 0.0584 & 0.2410 & 0.0356 & 0.0314 \\
\hline & \multirow{3}{*}{75} & $\vartheta$ & 0.0044 & 0.1275 & 0.0420 & 0.0208 & -0.0052 & 0.1139 & 0.0136 & 0.0141 & 0.0005 & 0.1320 & 0.0391 & 0.0208 \\
\hline & & $\varphi$ & 0.0107 & 0.0529 & -0.0142 & 0.0027 & 0.0394 & 0.1841 & -0.0441 & 0.0294 & 0.0177 & 0.1314 & -0.0412 & 0.0180 \\
\hline & & $\omega$ & -0.0409 & 0.0815 & 0.0144 & 0.0088 & 0.0139 & 0.1375 & 0.0544 & 0.0322 & 0.0160 & 0.0950 & 0.0033 & 0.0110 \\
\hline & \multirow{3}{*}{150} & $\vartheta$ & 0.0032 & 0.0758 & 0.0144 & 0.0054 & -0.0147 & 0.1033 & -0.0117 & 0.0114 & -0.0063 & 0.0875 & 0.0045 & 0.0082 \\
\hline & & $\varphi$ & 0.0068 & 0.0360 & -0.0067 & 0.0012 & 0.0141 & 0.1437 & -0.0432 & 0.0240 & -0.0090 & 0.1257 & -0.0387 & 0.0145 \\
\hline & & $\omega$ & -0.0118 & 0.0633 & 0.0107 & 0.0038 & 0.0116 & 0.1057 & 0.0410 & 0.0257 & 0.0280 & 0.0910 & 0.0257 & 0.0107 \\
\hline \multirow{9}{*}{1.4} & \multirow{3}{*}{35} & $\vartheta$ & -0.0058 & 0.1452 & 0.0568 & 0.0282 & -0.0144 & 0.1422 & 0.0191 & 0.0619 & -0.0823 & 0.1132 & -0.0425 & 0.0047 \\
\hline & & $\varphi$ & 0.0192 & 0.0731 & -0.0196 & 0.0050 & 0.1169 & 0.2848 & -0.0574 & 0.0906 & 0.4312 & 0.7019 & 0.0564 & 0.0312 \\
\hline & & $\omega$ & -0.0018 & 0.0481 & 0.0227 & 0.0037 & -0.0335 & 0.3389 & 0.0584 & 0.0900 & -0.0049 & 0.4563 & 0.0563 & 0.0158 \\
\hline & \multirow{3}{*}{75} & $\vartheta$ & 0.0103 & 0.1306 & 0.0400 & 0.0193 & 0.0158 & 0.1406 & 0.0315 & 0.0202 & 0.0076 & 0.1031 & -0.0302 & 0.0037 \\
\hline & & $\varphi$ & 0.0092 & 0.0504 & -0.0132 & 0.0026 & 0.0654 & 0.2036 & -0.0299 & 0.0333 & 0.0824 & 0.2920 & -0.0483 & 0.0893 \\
\hline & & $\omega$ & -0.0342 & 0.0443 & 0.0129 & 0.0507 & -0.0417 & 0.3233 & 0.0619 & 0.1163 & -0.0027 & 0.3049 & 0.0544 & 0.1258 \\
\hline & \multirow{3}{*}{150} & $\vartheta$ & -0.0013 & 0.0738 & 0.0179 & 0.0059 & 0.0012 & 0.0823 & 0.0182 & 0.0075 & 0.0028 & 0.0861 & 0.0214 & 0.0078 \\
\hline & & $\varphi$ & 0.0051 & 0.0347 & -0.0075 & 0.0012 & 0.0257 & 0.1288 & -0.0218 & 0.0143 & 0.0188 & 0.1428 & -0.0408 & 0.0248 \\
\hline & & $\omega$ & -0.0036 & 0.0403 & 0.0090 & 0.0011 & -0.0101 & 0.1849 & 0.0204 & 0.0287 & 0.0025 & 0.1398 & 0.0157 & 0.0253 \\
\hline \multirow{9}{*}{2.2} & \multirow{3}{*}{35} & $\vartheta$ & -0.0090 & 0.1432 & 0.0559 & 0.0278 & -0.0101 & 0.1617 & 0.0315 & 0.0244 & -0.0170 & 0.1386 & 0.0732 & 0.1433 \\
\hline & & $\varphi$ & 0.0201 & 0.0723 & -0.0187 & 0.0048 & 0.1400 & 0.2510 & -0.0249 & 0.0336 & 0.0643 & 0.2738 & -0.1272 & 0.3056 \\
\hline & & $\omega$ & -0.0017 & 0.0403 & 0.0140 & 0.0040 & -0.1369 & 0.4310 & 0.0343 & 0.0693 & 0.0476 & 0.3037 & 0.0332 & 0.1891 \\
\hline & \multirow{3}{*}{75} & $\vartheta$ & 0.0040 & 0.1178 & 0.0366 & 0.0166 & 0.0060 & 0.1182 & 0.0339 & 0.0156 & 0.0068 & 0.1310 & 0.0356 & 0.0191 \\
\hline & & $\varphi$ & 0.0088 & 0.0503 & -0.0133 & 0.0026 & 0.0396 & 0.1533 & -0.0368 & 0.0225 & 0.0583 & 0.2430 & -0.0427 & 0.0854 \\
\hline & & $\omega$ & -0.0258 & 0.0393 & 0.0252 & 0.0819 & -0.0340 & 0.3068 & 0.0532 & 0.1133 & -0.0061 & 0.2326 & 0.0490 & 0.1268 \\
\hline & \multirow{3}{*}{150} & $\vartheta$ & -0.0019 & 0.0730 & 0.0191 & 0.0061 & 0.0013 & 0.0760 & 0.0208 & 0.0068 & 0.0031 & 0.0865 & 0.0208 & 0.0078 \\
\hline & & $\varphi$ & 0.0049 & 0.0346 & -0.0075 & 0.0012 & 0.0119 & 0.1003 & -0.0270 & 0.0097 & 0.0354 & 0.1622 & -0.0288 & 0.0269 \\
\hline & & $\omega$ & 0.0008 & 0.0373 & 0.0043 & 0.0019 & -0.0016 & 0.1458 & 0.0169 & 0.0169 & -0.0133 & 0.1613 & 0.0050 & 0.0257 \\
\hline
\end{tabular}


Table 2: MLE and MPS estimation methods for XGExEx distribution with different values of parameters when $\vartheta=1.4$

\begin{tabular}{|c|c|c|c|c|c|c|c|c|c|c|c|c|c|c|}
\hline \multirow{2}{*}{\multicolumn{2}{|c|}{$\vartheta=1.4$}} & \multirow[t]{3}{*}{$\varphi$} & \multicolumn{4}{|c|}{0.5} & \multicolumn{4}{|c|}{1.4} & \multicolumn{4}{|c|}{2.2} \\
\hline & & & \multicolumn{2}{|c|}{ MLE } & \multicolumn{2}{|c|}{ MPS } & \multicolumn{2}{|c|}{ MLE } & \multicolumn{2}{|c|}{ MPS } & \multicolumn{2}{|c|}{ MLE } & \multicolumn{2}{|c|}{ MPS } \\
\hline$\omega$ & $\mathrm{n}$ & & Bias & MSE & Bias & MSE & Bias & MSE & Bias & MSE & Bias & MSE & Bias & MSE \\
\hline \multirow{9}{*}{0.5} & \multirow{3}{*}{35} & $\vartheta$ & 0.1116 & 0.5067 & 0.1764 & 0.3832 & -0.0749 & 0.4133 & -0.0030 & 0.1973 & -0.0543 & 0.4567 & 0.1313 & 0.2781 \\
\hline & & $\varphi$ & 0.0477 & 0.1445 & -0.0287 & 0.0135 & 0.0889 & 0.3480 & -0.1625 & 0.1379 & 0.0229 & 0.2918 & -0.1864 & 0.1713 \\
\hline & & $\omega$ & 0.0208 & 0.3303 & 0.1535 & 0.1599 & 0.0824 & 0.2909 & 0.2405 & 0.1906 & .1006 & 0.3209 & -0.0536 & 0.0737 \\
\hline & \multirow{3}{*}{75} & $\vartheta$ & 0.0577 & 0.2965 & 0.0781 & 0.0906 & -0.0276 & 0.3469 & -0.0063 & 0.1241 & 0.0288 & 4677 & 0.2344 & 0.2898 \\
\hline & & $\varphi$ & 0.0210 & 0.0875 & -0.0183 & 0.0060 & 0.0634 & 0.3157 & -0.1182 & 0.0940 & 0.0803 & .2743 & -0.0772 & 0.0730 \\
\hline & & $\omega$ & -0.0032 & 0.1959 & 0.0603 & 0.0436 & 0.0703 & 0.2531 & 0.1704 & 0.1214 & -0.0 & 0.2737 & 0.1094 & 936 \\
\hline & \multirow{3}{*}{150} & $\vartheta$ & -0.0220 & 0.2386 & 0.0038 & 0.0815 & -0.0438 & 0.2576 & -0.0522 & 0.0740 & 0.02 & .3064 & -0.0015 & \\
\hline & & $\varphi$ & 0.0124 & 0.0592 & -0.0108 & 0.0033 & 0.0069 & 0.19 & & & $0.0^{7}$ & & 751 & \\
\hline & & $\omega$ & 0.0049 & & & 0.0426 & 0.0 & & & & & & & \\
\hline \multirow{9}{*}{1.4} & \multirow{3}{*}{35} & $\vartheta$ & -0.0 & 0.4109 & & 288 & 0.0694 & & & & 0.0 & & & 327 \\
\hline & & $\varphi$ & 0.0413 & 0.1278 & -0.02 & 0.0136 & 0.2184 & 0.4 & & & 0.17 & & 837 & 0.3096 \\
\hline & & $\omega$ & 0.03 & & & 0.29 & -0.02 & & & & 0.0 & & & 739 \\
\hline & \multirow{3}{*}{75} & $\vartheta$ & 0.0398 & 0.3314 & & & 0.01 & 0.34 & & & 0.02 & & & 134 \\
\hline & & $\varphi$ & 0.0171 & 0.0834 & & 0.00 & & 0.2630 & -0.0 & & 34 & 0.4280 & -0.1663 & 0.2034 \\
\hline & & $\omega$ & -0.0313 & 0.3294 & 0.0483 & 0.1094 & -0.0014 & 0.2256 & & 752 & 0.0139 & 0.3839 & 0.1397 & 0.1981 \\
\hline & \multirow{3}{*}{150} & $\vartheta$ & -0.0168 & 0.1751 & 0.0371 & 0.0405 & 0.0357 & 0.3043 & -0.0098 & & -0.0017 & 0.2533 & 0.0636 & 0.0771 \\
\hline & & $\varphi$ & 0.0108 & 0.0560 & -0.0115 & 0.0031 & 0.1084 & 0.2173 & -0.0555 & 0.0742 & 0.0331 & 0.2446 & -0.0904 & 0.0579 \\
\hline & & $\omega$ & 0.0153 & 0.1920 & 0.0530 & 0.0459 & 0.0034 & 0.2048 & & 0.0703 & 0.0164 & 0.1721 & 0.0446 & 0.0283 \\
\hline \multirow{9}{*}{2.2} & \multirow{3}{*}{35} & $\vartheta$ & -0.0348 & 0.8984 & 0.1773 & 0.3371 & 0.0843 & 0.5793 & 0.1869 & 0.4680 & 0.0354 & 0.5607 & 0.2522 & 0.4787 \\
\hline & & $\varphi$ & 0.0397 & 0.1275 & -0.0289 & 0.0138 & 0.2332 & 0.4953 & -0.0914 & 0.1709 & 0.2000 & 0.6273 & -0.1701 & 0.2971 \\
\hline & & $\omega$ & 0.1350 & 0.9074 & 0.1636 & 0.2684 & -0.0454 & 0.5100 & 0.2529 & 0.4015 & -0.0350 & 0.4366 & 0.0967 & 0.1765 \\
\hline & \multirow{3}{*}{75} & $\vartheta$ & 0.0356 & 0.3245 & 0.1164 & 0.1372 & 0.0289 & 0.3679 & 0.0910 & 0.1495 & 0.0579 & 0.4205 & 0.1341 & 0.2126 \\
\hline & & $\varphi$ & 0.0169 & 0.0829 & -0.0213 & 0.0064 & 0.0803 & 0.2743 & -0.0772 & 0.0730 & 0.1787 & 0.4864 & -0.1209 & 0.2092 \\
\hline & & $\omega$ & -0.0315 & 0.3334 & 0.0549 & 0.1294 & -0.0182 & 0.2737 & 0.1094 & 0.0936 & -0.0380 & 0.3585 & 0.0856 & 0.1423 \\
\hline & \multirow{3}{*}{150} & $\vartheta$ & -0.0158 & 0.1774 & 0.0385 & 0.0386 & 0.0257 & 0.3064 & -0.0015 & 0.0903 & 0.0129 & 0.2767 & 0.0656 & 0.0810 \\
\hline & & $\varphi$ & 0.0107 & 0.0557 & -0.0115 & 0.0030 & 0.0751 & 0.2532 & -0.0536 & 0.0704 & 0.0643 & 0.2470 & -0.0787 & 0.0762 \\
\hline & & $\omega$ & 0.0113 & 0.1664 & 0.0485 & 0.0368 & 0.0129 & 0.2468 & 0.0923 & 0.0829 & -0.0242 & 0.2871 & 0.0692 & 0.0969 \\
\hline
\end{tabular}




\section{Application of Real Data Analysis}

This section is devoted to illustrate the potentiality of the XGExEx distribution for Two real data sets. XGExEx distribution is compared with other competitive models, namely: the Marshall-Olkin alpha power exponential (MOAPE) [Nassar et al. (2019)], extended odd Weibull exponential (EOWE) [Afify and Mohamed (2020)], WeibullExponential (WE) [Oguntunde et al. (2015)], ExEx and generalized exponential (GE) [Gupta and Kundu (2001)].

Tables 3, 4 provide values of Cram'er-von Mises ( $\left.W^{*}\right)$, AndersonDarling ( $\mathrm{A}^{*}$ ) and Kolmogorov- Smirnov (KS) statistic along with its Pvalue for the all models fitted based on Two real data sets. In addition, these tables contain the MLE and standard errors (SE) of the parameters for the considered models.

The first data represents a COVID-19 data belong to The United Kingdom of 76 days, from 15 April to 30 June 2020. these data formed of drought mortality rate. The data are as follows: 0.05870 .08630 .1165
$\begin{array}{llllllllll}0.1247 & 0.1277 & 0.1303 & 0.1652 & 0.2079 & 0.2395 & 0.2751 & 0.2845 & 0.2992\end{array}$
$\begin{array}{llllllllll}0.3188 & 0.3317 & 0.3446 & 0.3553 & 0.3622 & 0.3926 & 0.3926 & 0.4110 & 0.4633\end{array}$
$\begin{array}{llllllllll}0.4690 & 0.4954 & 0.5139 & 0.5696 & 0.5837 & 0.6197 & 0.6365 & 0.7096 & 0.7193\end{array}$
$\begin{array}{lllllllll}\mathbf{0 . 7 4 4 4} & \mathbf{0 . 8 5 9 0} & 1.0438 & 1.0602 & 1.1305 & 1.1468 & 1.1533 & 1.2260 & 1.2707\end{array}$
$\begin{array}{lllllllll}1.3423 & 1.4149 & 1.5709 & 1.6017 & 1.6083 & 1.6324 & 1.6998 & 1.8164 & 1.8392\end{array}$

$\begin{array}{lllllllll}1.8721 & 1.9844 & 2.1360 & 2.3987 & 2.4153 & 2.5225 & 2.7087 & 2.7946 & 3.3609\end{array}$

$\begin{array}{llllllllll}3.3715 & 3.7840 & 3.9042 & 4.1969 & 4.3451 & 4.4627 & 4.6477 & 5.3664 & 5.4500\end{array}$

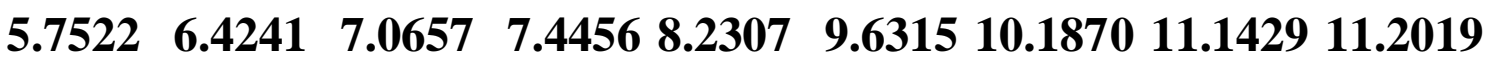
11.4584 .

Table 3. MLE, SE, KS test and P-values for COVID-19 data of The United Kingdom

\begin{tabular}{|c|c|c|c|c|c|c|c|}
\hline & & estimate & SE & $\mathrm{KS}$ & P-Value & $\mathrm{W}^{*}$ & $\mathrm{~A}^{*}$ \\
\hline \multirow{3}{*}{ XGExEx } & $\vartheta$ & 2.7766 & 2.1801 & \multirow{3}{*}{0.0604} & \multirow{3}{*}{0.9282} & \multirow{3}{*}{0.0727} & \multirow{3}{*}{0.5083} \\
\hline & $\varphi$ & 0.5275 & 0.1458 & & & & \\
\hline & $\omega$ & 0.6279 & 0.6680 & & & & \\
\hline \multirow{2}{*}{ ExEx } & $\varphi$ & 0.5839 & 0.1113 & \multirow{2}{*}{0.0617} & \multirow{2}{*}{0.9172} & \multirow{2}{*}{0.0744} & \multirow{2}{*}{0.5186} \\
\hline & $\omega$ & 1.1204 & 0.4511 & & & & \\
\hline \multirow{2}{*}{ GE } & $\beta$ & 0.8009 & 0.1171 & \multirow{2}{*}{0.0985} & \multirow{2}{*}{0.4245} & \multirow{2}{*}{0.1395} & \multirow{2}{*}{0.9287} \\
\hline & $\lambda$ & 0.3530 & 0.0569 & & & & \\
\hline \multirow{3}{*}{ WE } & $\alpha$ & 14.5701 & 16.5203 & \multirow{3}{*}{0.0806} & \multirow{3}{*}{0.6769} & \multirow{3}{*}{0.1196} & \multirow{3}{*}{0.8048} \\
\hline & $\beta$ & 0.8252 & 0.0751 & & & & \\
\hline & $\lambda$ & 0.0169 & 0.0207 & & & & \\
\hline \multirow{3}{*}{ EOWE } & $\alpha$ & 1.9148 & 0.6136 & \multirow{3}{*}{0.0672} & \multirow{3}{*}{0.8594} & \multirow{3}{*}{0.0751} & \multirow{3}{*}{0.5531} \\
\hline & $\beta$ & 8.8195 & 5.8230 & & & & \\
\hline & $\lambda$ & 1.5439 & 0.6527 & & & & \\
\hline \multirow{3}{*}{ MOAPE } & $\alpha$ & 0.0021 & 0.0012 & \multirow{3}{*}{0.0615} & \multirow{3}{*}{0.9189} & \multirow{3}{*}{0.0736} & \multirow{3}{*}{0.5421} \\
\hline & $\beta$ & 0.0322 & 0.0160 & & & & \\
\hline & $\lambda$ & 0.2553 & 0.1400 & & & & \\
\hline
\end{tabular}


مجلة البحوث المالية والتجارية ـ المجلد (22) ـ العدد الأول - يناير 2021

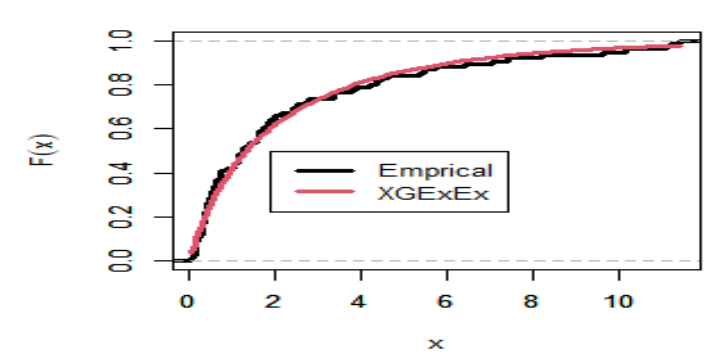

$Q-Q$ plot for $\times G E \times E x$

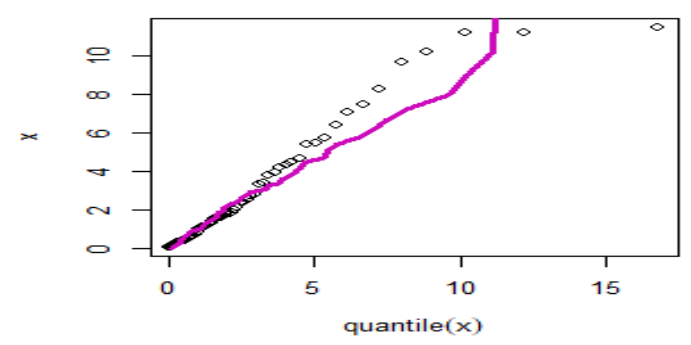

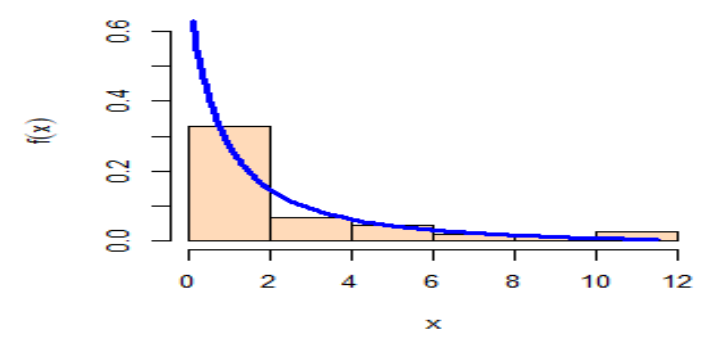

P-P plot for $\times G E \times E x$

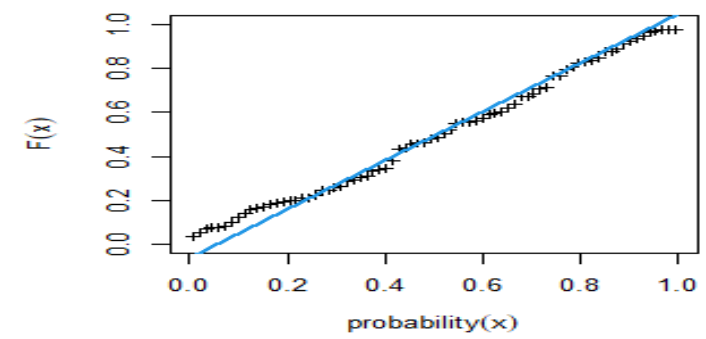

Figure 3. Cumulative function and empirical cdf, histogram and the Fitted XGExEx distribution, Q-Q plot and P-P plot for the XGExEx distribution for COVID-19 data of The United Kingdom.

The second data represents a COVID-19 data belong to Japan of 38 days, from 4 September to 12 October 2020. these data formed of drought mortality rate. The data are as follows: 0.15960 .27330 .1142 0.0851 0.1976 0.2243 0.1810 0.0828 0.1504 0.2169 0.0404 0.1208 0.1334 0.1589 0.1184 0.1698 0.0648 0.1027 0.0511 0.1019 0.1520 0.1006 0.0624

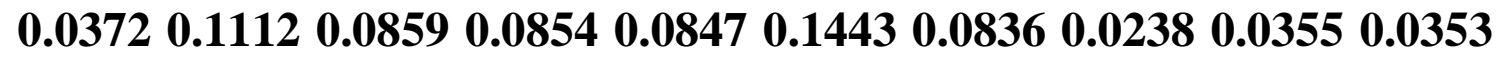
0.09370 .03490 .09240 .03440 .0228 .

Table 4. MLE, SE, KS test and P-values for COVID-19 data of Japan

\begin{tabular}{|c|c|c|c|c|c|c|c|}
\hline & & estimate & SE & $\mathrm{KS}$ & P-Value & $\mathrm{W}^{*}$ & $\mathrm{~A}^{*}$ \\
\hline \multirow{3}{*}{ XGExEx } & $\vartheta$ & 0.2339 & 0.0234 & \multirow{3}{*}{0.0805} & \multirow{3}{*}{0.9495} & \multirow{3}{*}{0.0415} & \multirow{3}{*}{0.3079} \\
\hline & $\varphi$ & 1.4372 & 0.0027 & & & & \\
\hline & $\omega$ & 41.7987 & 0.3739 & & & & \\
\hline \multirow{2}{*}{ ExEx } & $\varphi$ & 27.1401 & NA & \multirow{2}{*}{0.1961} & \multirow{2}{*}{0.0936} & \multirow{2}{*}{0.0460} & \multirow{2}{*}{0.3280} \\
\hline & $\omega$ & 0.2290 & NA & & & & \\
\hline \multirow{2}{*}{ GE } & $\beta$ & 3.2913 & 0.8781 & \multirow{2}{*}{0.1347} & \multirow{2}{*}{0.4558} & \multirow{2}{*}{0.0695} & \multirow{2}{*}{0.4558} \\
\hline & $\lambda$ & 17.7892 & 2.9010 & & & & \\
\hline \multirow{3}{*}{ WE } & $\alpha$ & 3.6476 & 7.1388 & \multirow{3}{*}{0.0930} & \multirow{3}{*}{0.8671} & \multirow{3}{*}{0.0461} & \multirow{3}{*}{0.3294} \\
\hline & $\beta$ & 1.5658 & 0.3052 & & & & \\
\hline & $\lambda$ & 2.9314 & 2.6984 & & & & \\
\hline \multirow{3}{*}{ EOWE } & $\alpha$ & 1.6494 & 0.3822 & \multirow{3}{*}{0.0956} & \multirow{3}{*}{0.8454} & \multirow{3}{*}{0.0433} & \multirow{3}{*}{0.3174} \\
\hline & $\beta$ & 0.4200 & 0.5177 & & & & \\
\hline & $\lambda$ & 6.3243 & 1.1032 & & & & \\
\hline \multirow{3}{*}{ MOAPE } & $\alpha$ & 25.0328 & 60.7498 & \multirow{3}{*}{0.0972} & \multirow{3}{*}{0.8314} & \multirow{3}{*}{0.0441} & \multirow{3}{*}{0.3324} \\
\hline & $\beta$ & 22.8634 & 5.5231 & & & & \\
\hline & $\lambda$ & 2.3743 & 3.1073 & & & & \\
\hline
\end{tabular}




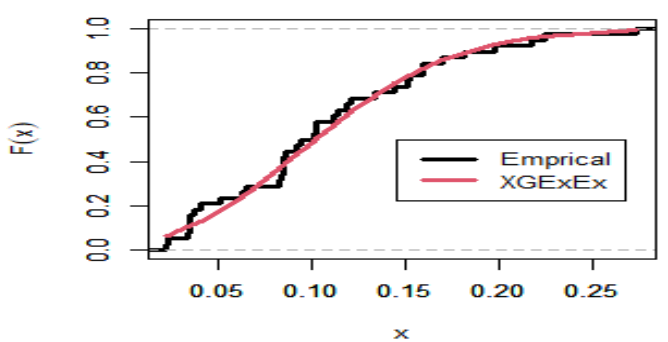

Q-Q plot for $\times$ GExEx

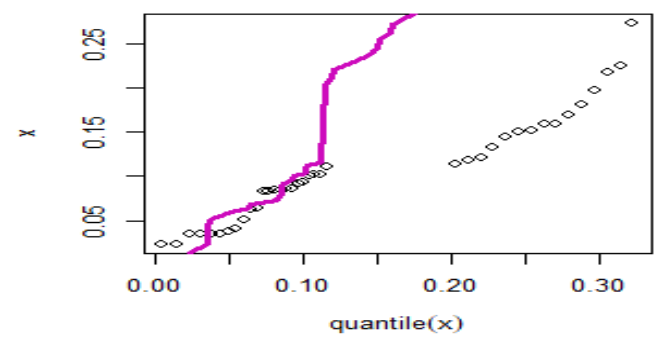

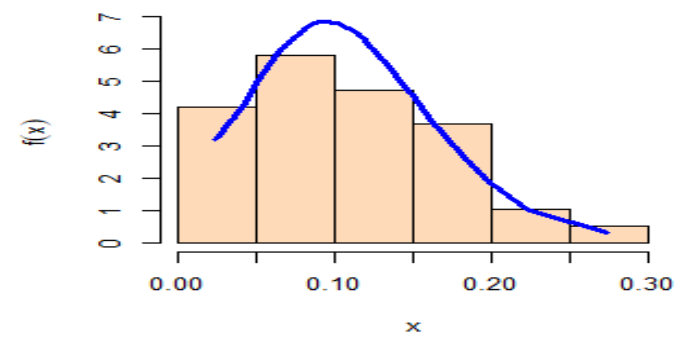

P-P plot for $\times$ GExEx

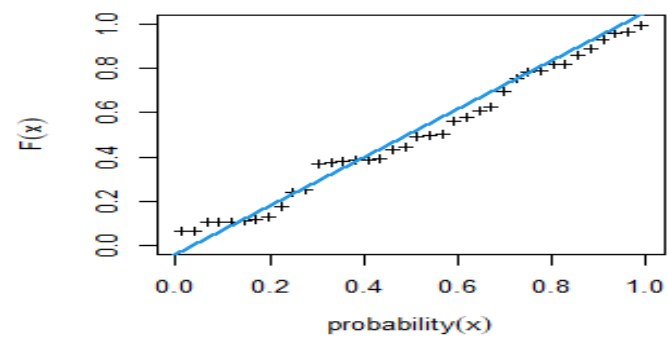

Figure 4. Cumulative function and empirical cdf, histogram and the Fitted XGExEx distribution, Q-Q plot and P-P plot for the XGExEx distribution for COVID-19 data of Japan.

In tables 3, 4, the XGExEx model has the highest $p$-value and the lowest distance (D) of $\operatorname{Kolmogorov-Smirnov}(\mathrm{K}-\mathrm{S}), \mathrm{W}^{*}$ and $\mathrm{A}^{*}$ value when it compares with all other models that used here to fit the COVID19 data. Figure 3,4 shows the fit empirical, histogram, QQ-plot and PPplot for the XGExEx distribution for COVID-19 data of the United Kingdom and Japan.

\section{Conclusion}

In this paper, we propose a new three-parameter distribution, called the X-Gamma extension of the exponential distribution which can be denoted as XGExEx distribution. The importance of the XGExEx distribution is derived from the wide utilization of the exponential model in life testing and the flexibility to analyze lifetime data. Survival and hazard functions of the XGExEx distribution are introduced. We provide some applications of XGExEx distribution in the context of statistics. The parameter estimation of XGExEx distribution is derived by MLE and MPS. The methods of estimation are employed to estimate the model parameters and simulation results are provided to evaluate the model performance. Two real-life data proposed model provides a consistently better fit than the ExEx, GE, WE, EOWE, MOAPE, and exponential distributions. Finally, we hope that the proposed model will be of great use in the application in various fields. 


\section{References:}

Afify, A. Z., \& Mohamed, O. A. (2020). A new three-parameter exponential distribution with variable shapes for the hazard rate: estimation and applications. Mathematics, 8(1), 135.

Almetwally, E. M., \&Almongy, H. M. (2019a). Estimation Methods for the New Weibull-Pareto Distribution: Simulation and Application. Journal of Data Science, 17(3), 610-630.

Almetwally, E. M., \&Almongy, H. M. (2019b). Maximum Product Spacing and Bayesian Method for Parameter Estimation for Generalized Power Weibull Distribution under Censoring Scheme. Journal of Data Science, 17(2), 407-444.

Almetwally, E. M., Almongy, H. M., \& Saleh, H. A. (2020). Managing Risk of Spreading" COVID-19" in Egypt: Modelling Using a Discrete Marshall-Olkin Generalized Exponential Distribution, International Journal of Probability and Statistics, 9(2), 33-41.

Almetwally, E. M., Gamal, M. I., (2020). Discrete Alpha Power Inverse Lomax Distribution with Application of COVID-19 Data, International Journal of Applied Mathematics, 9(6), 11-22.

Altun, E., \&Hamedani, G. G. (2018). The log-xgamma distribution with inference and application. Journal de la SociétéFrançaise de Statistique, 159(3), 40-55.

Ayyoubzadeh, S. M., Ayyoubzadeh, S. M., Zahedi, H., Ahmadi, M., \& Kalhori, S. R. N. (2020). Predicting COVID-19 incidence through analysis of google trends data in iran: data mining and deep learning pilot study. JMIR Public Health and Surveillance, 6(2), e18828.

Bantan, R., Hassan, A. S., Elsehetry, M., \&Kibria, B. M. (2020). HalfLogistic Xgamma Distribution: Properties and Estimation under Censored Samples. Discrete Dynamics in Nature and Society, 2020.

Biçer, H. D. (2019). Properties and inference for a new class of XGamma distributions with an application. Mathematical Sciences, 13(4), 335-346.

Caccavo, D. (2020). Chinese and Italian COVID-19 outbreaks can be correctly described by a modified SIRD model. medRxiv. DOI: 10.13140/RG.2.2.24485.86243.

Cheng, R. C. H., \& Amin, N. A. K. (1983). Estimating parameters in continuous univariate distributions with a shifted origin. Journal of the Royal Statistical Society. Series B (Methodological), 394-403. 
Cordeiro, G. M., Yousof, H. M.,Korkmaz, M. C.,PescimR. R., and Afify, A. Z.,(2019). The xgamma Family: Censored Regression Modellingand Applications. REVSTAT STATISTICAL JOURNAL, 1-22.

De Andrade, T. A., Bourguignon, M., \& Cordeiro, G. M. (2016). The exponentiated generalized extended exponential distribution. Journal of Data Science, 14(3), 393-413.

El-Morshedy, M., Eliwa, M. S., \& Nagy, H. (2020). A new twoparameter exponentiated discrete Lindley distribution: properties, estimation and applications. Journal of Applied Statistics, 47(2), 354375.

Gómez, Y. M., Bolfarine, H., \& Gómez, H. W. (2014). A new extension of the exponential distribution. Revista Colombiana de Estadística, 37(1), 25-34.

Gupta, R. D., \& Kundu, D. (2001). Generalized exponential distribution: different method of estimations. Journal of Statistical Computation and Simulation, 69(4), 315-337.

Hassan, A. S., \& Abd-Allah, M. (2018). ExponentiatedWeibull-Lomax distribution: properties and estimation. Journal of Data Science, 16(2), 277-298.

Hassan, A. S., Mohamd, R. E., Elgarhy, M., \& Fayomi, A. (2018). Alpha power transformed extended exponential distribution: properties and applications. Journal of Nonlinear Sciences and Applications, 12(4), 62-67.

Khan, M. S., King, R., \& Hudson, I. L. (2017). Transmuted generalized exponential distribution: $A$ generalization of the exponential distribution with applications to survival data. Communications in Statistics-Simulation and Computation, 46(6), 4377-4398.

Kumar, D., Dey, S., \& Nadarajah, S. (2017). Extended exponential distribution based on order statistics. Communications in StatisticsTheory and Methods, 46(18), 9166-9184.

Maleki, M., Mahmoudi, M. R., Wraith, D., \& Pho, K. H. (2020). Time series modelling to forecast the confirmed and recovered cases of COVID-19. Travel Medicine and Infectious Disease, 101742.

Muhammad, M. (2016). Poisson-odd generalized exponential family of distributions: theory and applications. Hacettepe Journal of Mathematics and Statistics, 47(6), 1652-1670. 
Nadarajah, S., \& Haghighi, F. (2011). An extension of the exponential distribution. Statistics, 45(6), 543-558.

Nassar, M., Kumar, D., Dey, S., Cordeiro, G. M., \& Afify, A. Z. (2019). The Marshall-Olkin alpha power family of distributions with applications. Journal of Computational and Applied Mathematics, 351, 41-53.

Oguntunde, P. E., Balogun, O. S., Okagbue, H. I., \& Bishop, S. A. (2015). The Weibull-exponential distribution: Its properties and applications. Journal of Applied Sciences, 15(11), 1305-1311.

Pena-Ramirez, F. A., Guerra, R. R., Cordeiro, G. M., \& Marinho, P. R. (2018). The exponentiated power generalized Weibull: Properties and applications. Anais da Academia Brasileira de Ciências, 90(3), 2553-2577.

Remuzzi, A., \& Remuzzi, G. (2020). COVID-19 and Italy: what next?. The Lancet 395(10231), 1225-1228. DOI: 10.1016/S01406736(20)30627-9.

Sen, S. and Chandra, S. S. N. (2017). The quasi xgamma distribution with application in bladder cancer data. Journal of data science, 15, 6176.

Sen, S., Al-Mofleh, H., \&Maiti, S. S. (2020). On discrimination between the Lindley and xgamma distributions. Annals of Data Science, 1-17.https://doi.org/10.1007/s40745-020-00243-7.

Sen, S., Chandra, N., \&Maiti, S. S. (2018a). On properties and applications of a two-parameter XGamma distribution. Journal of Statistical Theory and Applications, 17(4), 674-685.

Sen, S., Chandra, N., \&Maiti, S. S. (2018b). Survival estimation in xgamma distribution under progressively type-II right censored scheme. Model Assisted Statistics and Applications, 13(2), 107-121.

Sen, S., Maiti, S. S., \& Chandra, N. (2016). The xgamma distribution: statistical properties and application. Journal of Modern Applied Statistical Methods, 15(1), 38.

Yadav, A. S., Maiti, S. S., \&Saha, M. (2019). The inverse xgamma distribution: statistical properties and different methods of estimation. Annals of Data Science, 1-19.doi.org/10.1007/s40745-01900211-w. 